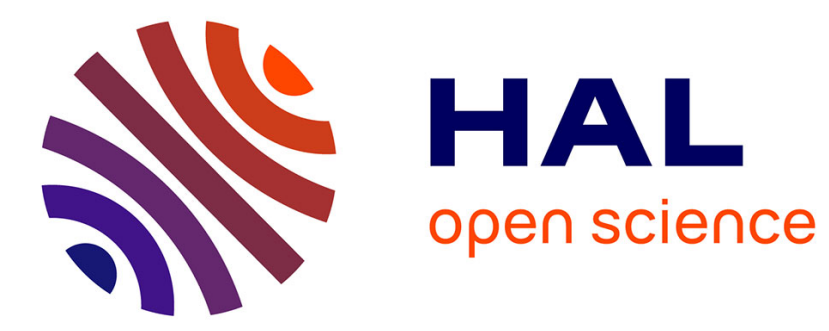

\title{
Les espaces de la coopération transfrontalière institutionnelle
}

François Moullé

\section{To cite this version:}

François Moullé. Les espaces de la coopération transfrontalière institutionnelle. Territoire en mouvement. Revue de Géographie et d'Aménagement, 2007, 4, pp.76 - 81. 10.4000/tem.921 . hal-03434409

\section{HAL Id: hal-03434409 \\ https://hal-univ-artois.archives-ouvertes.fr/hal-03434409}

Submitted on 18 Nov 2021

HAL is a multi-disciplinary open access archive for the deposit and dissemination of scientific research documents, whether they are published or not. The documents may come from teaching and research institutions in France or abroad, or from public or private research centers.
L'archive ouverte pluridisciplinaire $\mathbf{H A L}$, est destinée au dépôt et à la diffusion de documents scientifiques de niveau recherche, publiés ou non, émanant des établissements d'enseignement et de recherche français ou étrangers, des laboratoires publics ou privés. 
Territoire en mouvement Revue de géographie et aménagement

Territory in movement Journal of geography and planning

Santé et mobilité / Coopération transfrontalière

\section{Les espaces de la coopération transfrontalière institutionnelle}

The Spaces of Institutional Cross-Border Cooperation

\section{François Moullé}

\section{OpenEdition Journals}

Édition électronique

URL : https://journals.openedition.org/tem/921

DOI : $10.4000 /$ tem.921

ISSN : 1950-5698

\section{Éditeur}

Université des Sciences et Technologies de Lille

\section{Édition imprimée}

Date de publication : 1 octobre 2007

Pagination : 76-81

ISSN : 1954-4863

Ce document vous est offert par Université d'Artois

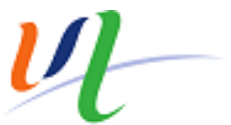

UNIVERSITÉ D'ARTOIS

Référence électronique

François Moullé, "Les espaces de la coopération transfrontalière institutionnelle », Territoire en mouvement Revue de géographie et aménagement [En ligne], 4 | 2007, mis en ligne le 15 décembre 2012, consulté le 18 novembre 2021. URL : http://journals.openedition.org/tem/921 ; DOI : https://doi.org/ 10.4000/tem.921

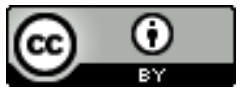

Territoire en mouvement est mis à disposition selon les termes de la licence Creative Commons Attribution 4.0 International 


\title{
Les espaces de la coopération transfrontalière institutionnelle
}

\author{
François MOULLE \\ Maître de Conférences en géographie \\ Université d'Artois \\ Laboratoire EA 2468 Dynamiques des réseaux \\ et des territoires \\ Maison de la Recherche \\ 9 rue du Temple BP 10665 \\ 62030 Arras Cedex \\ francois.moulle@univ-artois.fr
}

\begin{abstract}
Résumé
L'espace européen est complexe car les Etats n'ont pas toujours les mêmes degrés d'intégration territoriale. Ainsi, l'Union européenne ne correspond pas à l'espace Schengen ou encore à l'Union monétaire dite zone euro. Les frontières européennes n'ont donc pas la même qualité. Nonobstant cette diversité, la majorité des Etats a signé et ratifié la Convention de Madrid de 1981 en faveur de nouvelles coopérations transfrontalières. Les programmes Interreg, l'objectif $\mathrm{n}^{\circ} 3$ depuis le $1^{\text {er }}$ janvier 2007, permettent aux porteurs de projets de recevoir une participation financière de l'Union européenne. À partir de l'étude de dossiers Interreg, notamment pour les dyades franco-britannique et franco-belge, nous cherchons à définir les effets spatiaux de la coopération transfrontalière institutionnelle. De la phase 1 à la phase 3 du programme Interreg, les réseaux transfrontaliers produits ont évolué. Les porteurs de projets sont de plus en plus structurés. Les productions spatiales sont réelles, mais l'échelle locale, la plus fine, n'est pas la plus génératrice d'innovation spatiale sur le moyen terme.
\end{abstract}

Mots-clés : frontières, interreg, programme européen, coopération transfrontalière

La qualité juridique des dyades internes aux Etats membres de l'Union européenne n'est pas nécessairement homogène. Des différences notables existent selon les dyades étudiées. La coopération transfrontalière institutionnalisée par la Convention de Madrid et soutenue par le Programme Intégré Communautaire Interreg est à observer en interrelation avec les dimensions juridiques de la frontière. Les espaces transfrontaliers sont à la fois le fruit de dynamiques propres liées aux acteurs socioéconomiques locaux et régionaux, et des impulsions souhaitées et co-financées par l'Union européenne. Nous devons nous interroger sur les conséquences spatiales des impulsions euro76

\begin{abstract}
The European area is complicated because states lack the same degree of territorial integration. Thus, the European Union does not correspond to the Schengen area or monetary union called eurozone. European borders are not the same quality. Notwithstanding this diversity, the majority of states have signed and rattifié the Madrid Convention of 1981 for new cross-border cooperation. The Interreg, Objective number 3 from 1st Jan 2007 to allow project leaders to receive a financial contribution from the European Union. From the study of Interreg records, especially for dyads Franco-British and FrancoBelgian, we seek to define the spatial effects of crossborder institutions. Phase 1 to Phase 3 program Interreg cross-border networks products have evolved. The project leaders are increasingly structured. The productions are real space, but locally, the thinnest, is not the generator of space innovation over the medium term.
\end{abstract}

Key words: borders, interreg, european program, crossborder, cooperation

péennes. Pouvons-nous observer des productions spatiales originales dont la genèse serait due de manière explicite aux programmes mis en œuvre par la Commission européenne?

\section{LA COMPLEXITÉ DES ESPACES EUROPÉENS}

Les espaces frontaliers de l'Europe occidentale ne s'insèrent pas dans un espace géopolitique homogène même si l'Europe donne l'image d'unité et d'uniformité politiques vue des autres continents. Le processus d'intégration territoriale qui caractérise la construction européenne n'est ni linéaire dans le temps, ni uniforme dans l'espace. 
L'histoire a profondément marqué l'espace. Les Communautés européennes ont été initiées par six Etats. Ainsi, puisque nous étudions les relations transfrontalières franco-britanniques, la Grande-Bretagne n'a intégré les Communautés qu'en 1973 avec des droits négociés à l'époque qui ont encore des effets de nos jours. Contrairement aux derniers Etats intégrés de l'Europe centrale, la Grande-Bretagne n'a pas acquis l'ensemble du corpus juridique européen. C'est sur la base du volontariat et non de l'obligation qu'elle peut converger davantage vers ses partenaires. Cela explique en grande partie sa nonadhésion à des espaces spécifiques comme celui de l'Union monétaire et l'espace Schengen.

De nos jours, le premier calque spatial de l'Europe est l'Union européenne à 27 Etats membres. Il est complété par l'Espace Economique Européen (EEE) intégrant des pays comme l'Islande et la Norvège. Le statut d'Etat EEE a été proposé aux anciens membres de l'Association Européenne de Libre Echange (AELE) pour s'intégrer dans un espace économique ouvert avec des règles en convergence sans pour autant participer à la dimension politique de l'Union européenne. L'AELE a perdu une partie de son sens dès l'intégration de la Grande-Bretagne dans les Communautés européennes, car elle avait le rôle de leader de l'AELE, créée en réaction au traité de Rome. Parmi les membres de l'AELE qui n'ont pas été volontaires pour faire partie de l'Union européenne, seule la Suisse a refusé, en 1992, les règles de l'EEE. L'Union Européenne Monétaire (UEM) a été, à l'origine, mise en place dans le cadre d'un partenariat intergouvernemental. Seuls les Etats volontaires ont souscrit à la démarche qui aboutira à la monnaie unique, l'Euro. Cette démarche pleinement politique a joué le rôle d'un différenciateur à l'époque de l'Europe des douze. C'est ainsi que la Grande-Bretagne et l'Irlande ont échappé à une convergence qu'elles ne souhaitaient pas.

Comme pour l'Union européenne, les négociations pour un espace de liberté des personnes ont débuté en 1985 de manière intergouvernementale. C'est pourquoi, lors de sa mise en place en 1995, l'espace Schengen ne correspond pas à l'Union européenne. La Grande-Bretagne échappe à nouveau à un des éléments originaux de la convergence territoriale européenne.
Le résultat est une Europe à géométrie variable. Le noyau est constitué par les Etats initiateurs du processus général à la fin des années cinquante et progressivement accompagné par les nouveaux Etats membres de l'Union européenne intégrés depuis 1992 qui ont l'obligation d'adhérer à terme à la totalité du corpus juridique européen. Ils adhèrent donc de fait aux différents calques spatiaux qui caractérisent la complexité européenne.

Les frontières étudiées à travers le programme Interreg ne sont donc pas semblables. Le détroit du Pas de Calais est une discontinuité physique à la morphologie bien identifiée. Les traits de côtes constituent des limites perceptibles même si le tunnel sous la Manche a perturbé partiellement la perception des choses. Les frontières franco-britannique et anglo-belge sont des discontinuités internes à l'Union européenne. Mais elles constituent des frontières externes pour l'Union monétaire et pour l'espace Schengen. La monnaie est différente de part et d'autre de la Manche. Surtout, les règles de circulation des personnes changent avec un contrôle aux frontières qui s'est renforcé avec la mise en place du Système Schengen. Le nombre de réfugiés sur les côtes françaises en attente d'un hypothétique passage clandestin vers la Grande-Bretagne en est un bon révélateur. L'enfermement des quais pour Londres à la gare du Nord est une dimension visible de la sécurisation de la frontière externe de l'espace Schengen.

Par contre, la frontière franco-belge est un modèle pour la logique d'intégration des territoires européens. Les deux Etats font partie des mêmes calques spatiaux européens. La monnaie commune et la dématérialisation des contrôles sur la ligne frontière permettent un passage aisé. Seuls les vestiges des postes douaniers rappellent aux citoyens les plus attentifs l'époque des contrôles douaniers.

\section{LES POLITIQUES EUROPÉENNES EN FAVEUR D'UNE COOPÉRATION TERRI. TORIALE}

L'objectif n'est pas de présenter en détail les politiques européennes mais de comprendre le processus mis en place en faveur de la coopération entre des territoires européens. 
Les années soixante-dix sont marquées par une série de rencontres européennes soulignant l'absence de cohérence entre un projet européen unificateur et des Etats limitant les relations transfrontalières à des relations interEtatiques. Il faut attendre 1981 pour que soit signée par les Etats membres des Communautés Européennes et certains Etats de l'Association Européenne de Libre Echange (AELE) la Convention de Madrid. La ratification par chaque Etat a été un processus long, d'autant que des accords additionnels ont suivi. Par exemple, pour la France, il a fallu attendre que le processus de décentralisation engagé par les lois de 1982 soit suffisamment avancé pour être en cohérence avec les engagements de la Convention de Madrid. L'Italie n'a toujours pas ratifié la totalité des accords additionnels. Enfin, la Suisse qui ne s'insère pas dans un des espaces européens, a été un des Etats à ratifier l'ensemble de la Convention. Cette diversité révèle des cultures politiques et institutionnelles différentes. La Convention de Madrid a une logique intergouvernementale.

En 1991, la Commission européenne sise à Bruxelles met en place un Programme Intégré Communautaire (PIC) pour accompagner les territoires frontaliers vers les objectifs de la Convention de Madrid, c'est-à-dire la mise en place de coopérations transfrontalières favorisant les convergences et les complémentarités territoriales. Les espaces transfrontaliers sont dès lors considérés comme des espaces expérimentaux pour l'intégration européenne en général. Les marges des Etats deviennent paradoxalement le cour de l'esprit de convergence. Le retard, en matière de développement des marges lié à l'histoire de l'Europe jusqu'au milieu du $\mathrm{XX}^{\mathrm{e}}$ siècle, doit ainsi être rattrapé par des subventions ciblées. Le PIC porte le nom d'Interreg (Interrégional) et fonctionne dans une logique de subsidiarité. Les axes prioritaires sont fixés par les collectivités territoriales régionales. La gestion doit se faire au plus près des porteurs de projets. Ces porteurs peuvent être des collectivités, des entreprises, des associations et toutes formes de regroupements sociaux reconnus par la loi. Le porteur de projet doit à la fois apporter la preuve d'une capacité financière au moins égale à la moitié des 78 besoins du projet et avoir une trésorerie suffisante pour faire l'avance des aides européennes puisqu'une partie des subventions tombe une fois le projet réalisé.

Interreg a été développé en trois phases : 1990. 1993 ; 1994-1999; 2000-2006.

La première phase peut être qualifiée d'expérimentale. Les porteurs de projets avaient des dossiers relativement légers à instruire. Le partenaire au-delà de la frontière était simplement indiqué. Par contre, les lignes budgétaires étaient plutôt modestes.

À partir de la deuxième phase, un accord de partenariat avec un ou plusieurs partenaires transfrontaliers était un prérequis indispensable. Cela veut dire que les objectifs et les moyens à mettre en ouvre devaient être définis préalablement à l'instruction du dossier. Par ailleurs, une réflexion des porteurs des projets sur les outils d'évaluation nécessaires à mettre en oeuvre devait être présentée. Autant dire que le dossier, bien que national, était plus complexe.

Enfin, la troisième phase exigeait des porteurs de projets un dossier détaillé et unique par partenariat.

Nous constatons donc trois caractéristiques pour le programme interreg:

- Des phases de plus en plus longues permettant des projets de plus en plus ambitieux,

- Des budgets croissants,

- Des dossiers de demande de subvention de plus en plus complexes à instruire.

Bien entendu, les trois phases n'ont pas donné les mêmes résultats en termes spatiaux.

La Commission européenne a proposé une nouvelle architecture pour la politique de cohésion 2007-2013 de l'Union. Elle repose sur les thèmes liés à la stratégie définie par les Conseils Européens de Lisbonne en 2000 et Göteborg en 2001.

La nouvelle politique de cohésion s'articule autour de trois grands objectifs prioritaires. Nous ne nous étendrons pas sur la "Convergence » pour les régions d'outre-mer ni sur la «Compétitivité régionale et l'emploi ». Par contre, l'innovation pour notre domaine d'étude est celui de l'objectif 3 : la «Coopération européenne ». 
Cet objectif reprend les trois volets de l'actuel programme Interreg III :

- la coopération transfrontalière, le long des frontières terrestres et maritimes ;

- la coopération transnationale sur des priorités stratégiques (recherche, société de l'information, environnement...) ;

- la coopération interrégionale permettant aux régions de coopérer en réseau à l'échelle de l'Europe, de mettre en place des réseaux de coopération et de favoriser les échanges d'expériences.

L'innovation à partir de 2007 est bien de mettre la priorité sur la coopération territoriale européenne. Jusqu'ici, Interreg a permis la construction d'espaces et de réseaux.

\section{LES ESPACES DE LA COOPÉRATION TRANSFRONTALIÈRE}

Létude scientifique constituant le cœur de ce numéro thématique de Territoire en Mouvement est l'analyse des dossiers Interreg. La définition d'un espace est liée à la localisation des porteurs de projets.

Les espaces qui se dégagent des coopérations institutionnelles transfrontalières de la période
Interreg 1 sont très variés. À l'échelle locale, de nombreux partenariats constituent un chapelet de micros espaces, de part et d'autre des lignes frontières. Parfois, ces espaces sont relativement éloignés $(20 / 50 \mathrm{~km})$ de la ligne frontière. Paradoxalement, le détroit du Pas-de-Calais ne constitue pas en lui-même un facteur de différenciation spatiale entre l'espace franco-britannique et l'espace franco-belge. La discontinuité maritime, notamment sur la période Interreg 1, n'a pas été perçu par les porteurs de projets comme une contrainte spécifique.

Les porteurs de projets ont des statuts variés, même si les communes dominent.

À une échelle supérieure, nous trouvons de manière forte les collectivités territoriales : Wallonie et Flandre, les Provinces Belges, les Comtés Britanniques, les Départements et les Régions Françaises. Bien entendu, apparaissent aussi des regroupements de collectivités locales notamment sur la dyade franco-belge. À cette échelle, des espaces sont aussi animés par des Unions professionnelles. Notons que ces espaces de coopérations se caractérisent par des relations sociales entre des lieux, leurs structures sont le plus souvent constituées en réseaux.

Figure 1 : Une multitude de porteurs de projet à l'échelle locale

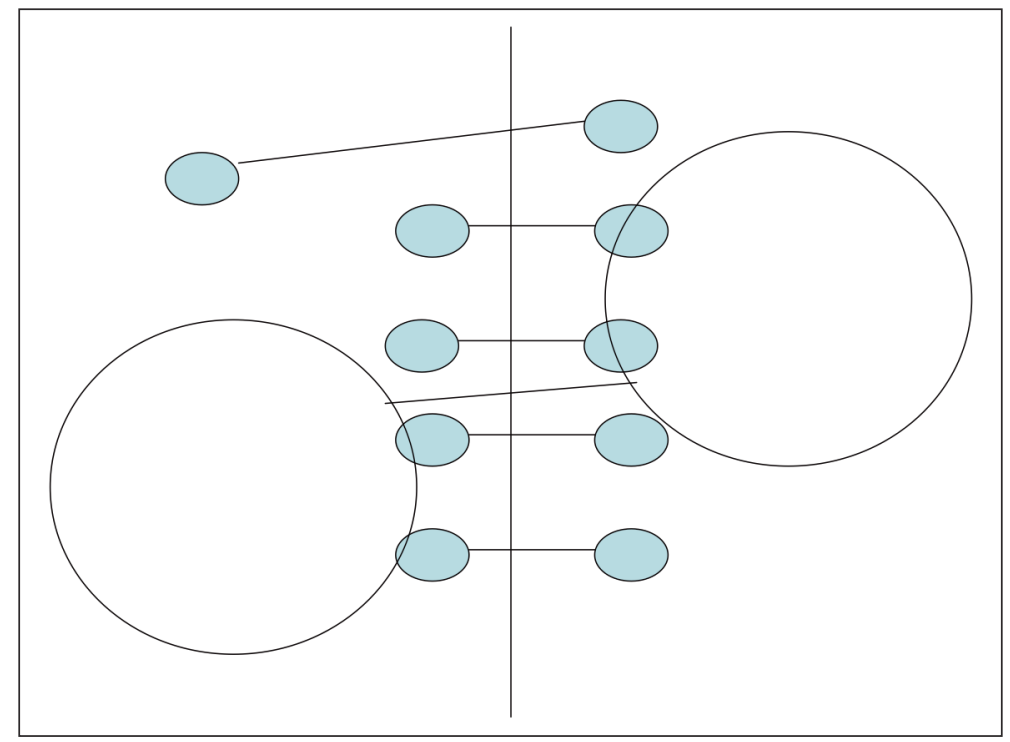

Interreg 2, du fait de sa complexité croissante, voit l'importance des micros espaces diminuer au profit des espaces d'importances régionales. Néanmoins, cette phase est marquée par le développement très net de réalisations concrètes qui prennent la forme d'espaces en miroirs, des réalisations identiques apparaissent de part et d'autre de la frontière. Les collectivités territoriales les plus importantes sont celles qui portent le plus de projets. Nous observons donc l'absorption progressive des micros espaces aux profits d'espaces d'échelles plus petites. 
Figure 2: Avec Interreg 2, les initiatives sont plus centralisées

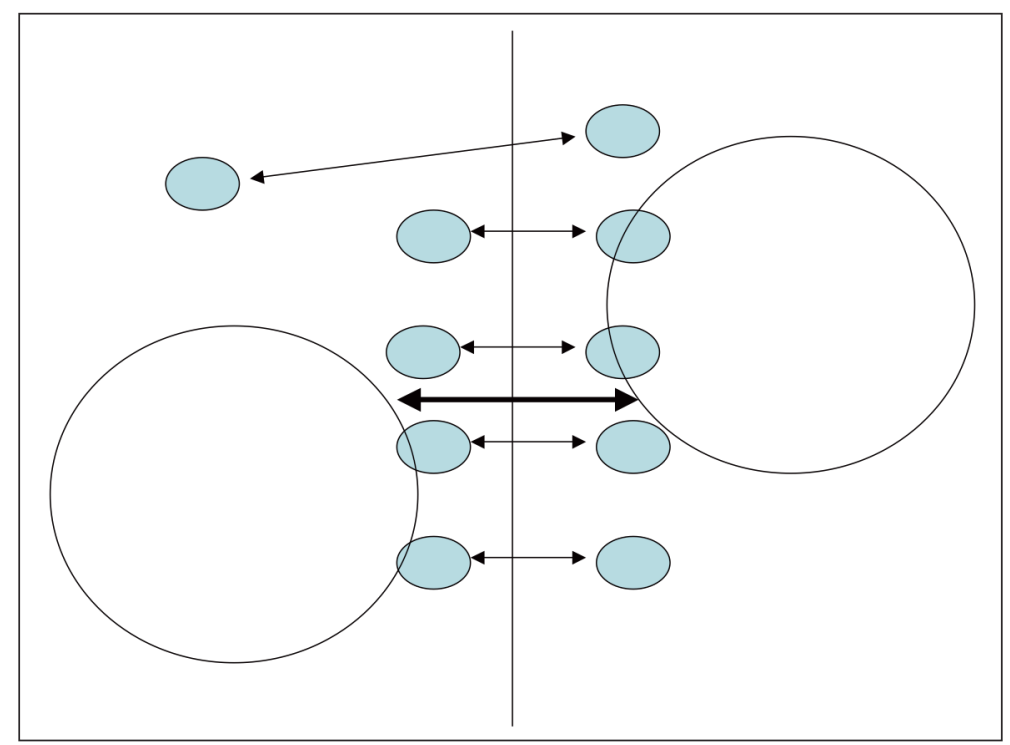

Enfin, Interreg 3 marginalise clairement les petits porteurs de projets. La technicité des dossiers a tendance à éliminer les petites collectivités, les associations modestes et les entreprises aux moyens limités. D'une certaine manière, les trois phases Interreg révèlent l'importance croissante des moyens nécessaires pour participer à la coopération transfrontalière. Progressivement, ce sont les collectivités locales les plus importantes et les regroupements professionnels qui remportent les subventions car instruire les dossiers nécessite aujourd'hui une véritable ingénierie.

Figure 3 : La domination des collectivités territoriales supérieures dans le cadre d'Interreg 3

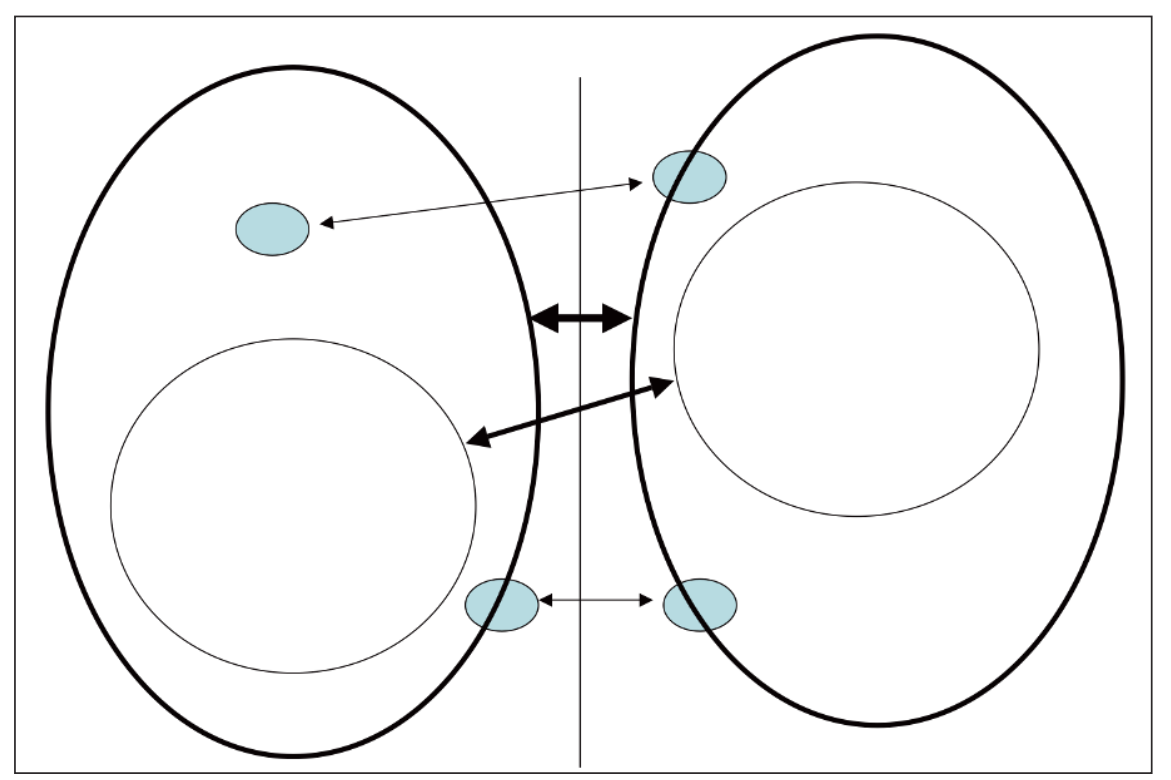

Létude détaillée des dossiers Interreg permet de souligner la mise en place de marqueurs spatiaux nouveaux et originaux. Les trois phases d'Interreg ne favorisent pas la genèse d'espaces à des échelles identiques. Initialement, les productions locales sont les plus nombreuses mais les moins matérialisées. Progressivement, les porteurs de projets sont socialement et éco80 nomiquement plus solides. La perte en finesse scalaire est compensée par une concrétisation plus importante des projets. Par ailleurs, d'une dyade à l'autre, les situations sont différentes, dues en partie à des variations juridiques et à des différences culturelles évidentes.

Les espaces produits sont pour une majorité, réticulaires avec l'apparition, soutenus par les 
subventions Interreg, de réseaux transfrontaliers très diversifiés où les institutions et les personnes jouent un rôle central. Par exemple, dans les domaines de la formation et de la culture, la densité des réseaux constitue de nouveaux espaces véritablement transfrontalier. Même dans le cadre industriel, ce sont des réseaux d'entreprises qui participent au programme Interreg. C'est particulièrement vrai pour la dyade franco-belge.

Ces productions réticulaires ne remettent pas en cause les espaces de pouvoir antérieurs et en positions frontalières, par contre elles complexifient nettement la gouvernance transfron- talière qui doit aujourd'hui prendre en compte la vitalité des espaces réticulaires et le rôle important des collectivités territoriales

La frontière, qui par discontinuité met de la distance dans la proximité (Arbaret-Schulz, 2002), produit actuellement des réseaux qui tendent à créer de nouvelles proximités.

Interreg impulse donc de l'innovation spatiale car la frontière devient rééllement objet de gestion et non de confrontation (cf Dayez, Leloup, Verger, Du problème frontalier au projet transfrontalier : vers une gouvernance territoriale?). Cette innovation constitue un aspect de l'intégration européenne.

\section{Bibliographie}

ARBARET-SCHULZ Ch., « les villes européennes, attracteurs étranges de formes frontalières nouvelles », in Bernard Reitel, Patricia Zander, Jean-Luc Piermay, Jean-Pierre Renard, Villes et frontières, Anthropos-Economica, Collection Villes, 2002, p. 213-230.

COMTE H., LEVRAT N. (dir.), 2006, Aux coutures de l'Europe : défis et enjeux juridiques de la coopération transfrontalière, Paris, l'Harmattan, 365 p.

Groupe FRONTIÈRE, Christiane ARBARETSCHULZ, Antoine BEYER, Jean-Luc PIERMAY, Bernard REITEL, Catherine SELIMANOVSKI, Christophe SOHN et Patricia ZANDER, "La frontière, un objet spatial en mutation."

EspacesTemps.net, Textuel, 29.10.

2004http://espacestemps.net/document842.html
LÉVY J., 1997, Europe, Une géographie, Hachette sup., $288 \mathrm{p}$.

MOULLÉ F. (coord.), 2003, La Frontière Nord, in Fourny-kober M.C (coordination), Le rapport à la frontière et la construction d'espaces transfrontaliers, DATAR-programme de recherche Prospectives 2020, 63 p.

PEYRONY J. et alii, 2002, Le schéma de développement de l'espace communautaire, Paris, La Documentation française, $103 \mathrm{p}$.

RENARD J.P. (dir.), 2002, « La frontière : limite politique majeure, mais aussi aire de transition », in Collectif, Limites et discontinuités en géographie, Paris, Sedes, p. 40-66.

2007, Atlas de la coopération transfrontalière, Mission Opérationnelle Transfrontalière, $2^{\mathrm{e}}$ éd. 